University of Texas at El Paso

ScholarWorks@UTEP

$12-2014$

\title{
Interval computations and interval-related statistical techniques: estimating uncertainty of the results of data processing and indirect measurements
}

Vladik Kreinovich

The University of Texas at El Paso, vladik@utep.edu

Follow this and additional works at: https://scholarworks.utep.edu/cs_techrep

Part of the Computer Engineering Commons

Comments:

Technical Report: UTEP-CS-14-74

Published in: Franco Pavese (ed.), Advanced Mathematical and Computational Tools in

Metrology and Testing AMTCM'X, World Scientific, Singapore, 2015, pp. 38-49.

\section{Recommended Citation}

Kreinovich, Vladik, "Interval computations and interval-related statistical techniques: estimating uncertainty of the results of data processing and indirect measurements" (2014). Departmental Technical Reports (CS). 875.

https://scholarworks.utep.edu/cs_techrep/875

This Article is brought to you for free and open access by the Computer Science at ScholarWorks@UTEP. It has been accepted for inclusion in Departmental Technical Reports (CS) by an authorized administrator of ScholarWorks@UTEP.For more information, please contact Iweber@utep.edu. 
Interval computations and interval-related statistical techniques: estimating uncertainty of the results of data processing and indirect measurements

\author{
V. Kreinovich \\ Computer Science Department, University of Texas at El Paso, \\ El Paso, Texas 79968, USA \\ E-mail: vladik@utep.edu \\ http://www.cs.utep.edu/vladik
}

\begin{abstract}
In many practical situations, we only know the upper bound $\Delta$ on the measurement error: $|\Delta x| \leq \Delta$. In other words, we only know that the measurement error is located on the interval $[-\Delta, \Delta]$. The traditional approach is to assume that $\Delta x$ is uniformly distributed on $[-\Delta, \Delta]$. In some situations, however, this approach underestimates the error of indirect measurements. It is therefore desirable to directly process this interval uncertainty. Such "interval computations" methods have been developed since the 1950s. In this paper, we provide a brief overview of related algorithms and results.
\end{abstract}

Keywords: interval uncertainty, interval computations, interval-related statistical techniques

\title{
1. Need for Interval Computations
}

Data processing and indirect measurements. We are often interested in a physical quantity $y$ that is difficult (or impossible) to measure directly: distance to a star, amount of oil in a well. A natural idea is to measure $y$ indirectly: we find easier-to-measure quantities $x_{1}, \ldots, x_{n}$ related to $y$ by a known relation $y=f\left(x_{1}, \ldots, x_{n}\right)$, and then use the results $\widetilde{x}_{i}$ of measuring $x_{i}$ to estimate $\widetilde{y}$ :

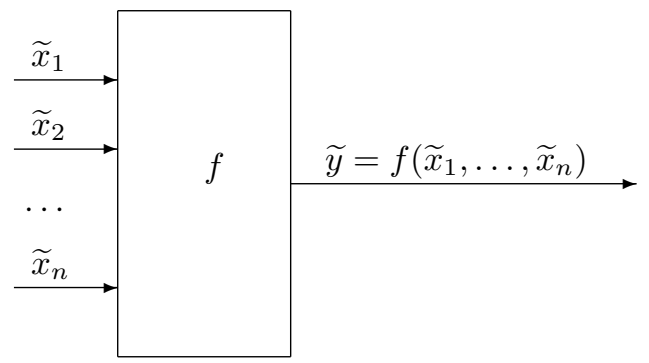


This is known as data processing.

Estimating uncertainty of the results of indirect measurements: a problem. Measurements are never $100 \%$ accurate. The actual value $x_{i}$ of $i$-th measured quantity can differ from the measurement result $\widetilde{x}_{i}$; in other words, there are measurement errors $\Delta x_{i} \stackrel{\text { def }}{=} \widetilde{x}_{i}-x_{i}$. Because of that, the result $\widetilde{y}=f\left(\widetilde{x}_{1}, \ldots, \widetilde{x}_{n}\right)$ of data processing is, in general, different from the actual value $y: \widetilde{y}=f\left(\widetilde{x}_{1}, \ldots, \widetilde{x}_{n}\right) \neq f\left(x_{1}, \ldots, x_{n}\right)=y$. It is desirable to describe the error $\Delta y \stackrel{\text { def }}{=} \widetilde{y}-y$ of the result of data processing. For this, we must have information about the errors of direct measurements.

Uncertainty of direct measurements: need for overall error bounds (i.e., interval uncertainty). Manufacturers of a measuring instrument (MI) usually provide an upper bound $\Delta_{i}$ for the measurement error: $\left|\Delta x_{i}\right| \leq \Delta_{i}$. (If no such bound is provided, then $\widetilde{x}_{i}$ is not a measurement, it is a wild guess.)

Once we get the measured value $\widetilde{x}_{i}$, we can thus guarantee that the actual (unknown) value of $x_{i}$ is in the interval $\mathbf{x}_{i} \stackrel{\text { def }}{=}\left[\widetilde{x}_{i}-\Delta_{i}, \widetilde{x}_{i}+\Delta_{i}\right]$. For example, if $\widetilde{x}_{i}=1.0$ and $\Delta_{i}=0.1$, then $x_{i} \in[0.9,1.1]$.

In many practical situations, we also know the probabilities of different values $\Delta x_{i}$ within this interval. It is usually assumed that $\Delta x_{i}$ is normally distributed with 0 mean and known standard deviation.

In practice, we can determine the desired probabilities by calibration, i.e., by comparing the results $\widetilde{x}_{i}$ of our MI with the results $\widetilde{x}_{i}{ }^{\text {st }}$ of measuring the same quantity by a standard (much more accurate) MI. However, there are two cases when calibration is not done: (1) cutting-edge measurements (e.g., in fundamental science), when our MI is the best we have, and (2) measurements on the shop floor, when calibration of MI is too expensive.

In both cases, the only information we have is the upper bound on the measurement error. In such cases, we have interval uncertainty about the actual values $x_{i}$; see, e.g., ${ }^{11}$.

Interval computations: a problem. When the inputs $x_{i}$ of the data processing algorithms are known with interval uncertainty, we face the following problem:

- Given: an algorithm $y=f\left(x_{1}, \ldots, x_{n}\right)$ and $n$ intervals $\mathbf{x}_{i}=\left[\underline{x}_{i}, \bar{x}_{i}\right]$.

- Compute: the corresponding range of $y$ :

$$
[\underline{y}, \bar{y}]=\left\{f\left(x_{1}, \ldots, x_{n}\right) \mid x_{1} \in\left[\underline{x}_{1}, \bar{x}_{1}\right], \ldots, x_{n} \in\left[\underline{x}_{n}, \bar{x}_{n}\right]\right\} .
$$




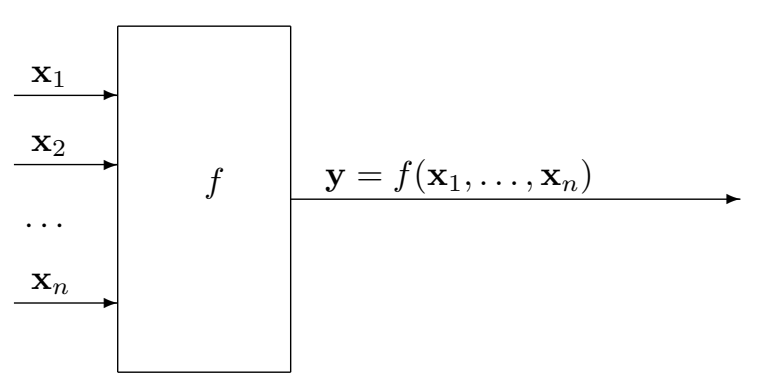

It is known that this problem is NP-hard even for quadratic $f$; see, e.g., ${ }^{8}$. In other words, unless $\mathrm{P}=\mathrm{NP}$ (which most computer scientists believe to be impossible), no feasible algorithm is possible that would always compute the exact range $\mathbf{y}$. We thus face two major challenges: (1) find situations feasible algorithms are possible, and (2) in situations when the exact computation of $\mathbf{y}$ is not feasibly possible, find feasible algorithms for computing a good approximation $\mathbf{Y} \supseteq \mathbf{y}$.

\section{Alternative Approach: Maximum Entropy (MaxEnt)}

Idea: a brief reminder. Traditional engineering approach to uncertainty is to use probablistic techniques, based on probability density functions (pdf) $\rho(x)$ and cumulative distribution functions (cdf) $F(x) \stackrel{\text { def }}{=} P(X \leq x)$. As we have mentioned, in many practical applications, it is very difficult to come up with the probabilities. In such applications, many different probability distributions are consistent with the same observations. In such situations, a natural idea is to select one of these distributions - e.g., the one with the largest entropy $S \stackrel{\text { def }}{=}-\int \rho(x) \cdot \ln (\rho(x)) d x$; see, e.g., ${ }^{5}$.

Often, this idea works. This approach often leads to reasonable results. For example, for the case of a single variable $x$, if all we know is that $x \in[\underline{x}, \bar{x}]$, then MaxEnt leads to a uniform distribution on $[\underline{x}, \bar{x}]$. For several variables, if we have no information about their dependence, MaxEnt implies that different variables are independently distributed.

Sometimes, this idea does not work. Sometimes, the results of MaxEnt are misleading. As an example, let us consider the simplest algorithm $y=x_{1}+\ldots+x_{n}$, with $\Delta x_{i} \in[-\Delta, \Delta]$. In this case, $\Delta y=\Delta x_{1}+\ldots+\Delta x_{n}$. The worst case is when $\Delta_{i}=\Delta$ for all $i$, then $\Delta y=n \cdot \Delta$.

What will MaxEnt return here? If all $\Delta x_{i}$ are uniformly distributed, then for large $n$, due to the Central Limit Theorem, $\Delta y$ is approximately 
normal, with $\sigma=\Delta \cdot \frac{\sqrt{n}}{\sqrt{3}}$.

With confidence $99.9 \%$, we can thus conclude that $|\Delta y| \leq 3 \sigma$; so, we get $\Delta \sim \sqrt{n}$, but, as we mentioned. it is possible that $\Delta=n \cdot \Delta \sim n$ which, for large $n$, is much larger than $\sqrt{n}$.

The conclusion from this example is that using a single distribution can be very misleading, especially if we want guaranteed results - and we do want guaranteed results in high-risk application areas such as space exploration or nuclear engineering.

\section{Possibility of Linearization}

Linearization is usually possible. Each interval has the form $\left[\widetilde{x}_{i}-\Delta_{i}, \widetilde{x}_{i}-\Delta_{i}\right]$, where $\widetilde{x}_{i}$ is a midpoint and $\Delta_{i}$ is half-width. Possible values $x_{i}$ are $x_{i}=\widetilde{x}_{i}+\Delta x_{i}$, with $\left|\Delta x_{i}\right| \leq \Delta_{i}$, so $f\left(x_{1}, \ldots, x_{n}\right)=$ $f\left(\widetilde{x}_{1}+\Delta x_{1}, \ldots, \widetilde{x}_{n}+\Delta x_{n}\right)$. The values $\Delta_{i}$ are usually reasonable small, hence the values $\Delta x_{i}$ are also small. Thus, we can expand $f$ into Taylor series and keep only linear terms in this expansion:

$$
f\left(\widetilde{x}_{1}+\Delta x_{1}, \ldots\right)=\widetilde{y}+\sum_{i=1}^{n} c_{i} \cdot \Delta x_{i} \text {, where } \widetilde{y} \stackrel{\text { def }}{=} f\left(\widetilde{x}_{1}, \ldots\right) \text { and } c_{i} \stackrel{\text { def }}{=} \frac{\partial f}{\partial x_{i}} .
$$

Here, $\max \left(c_{i} \cdot \Delta x_{i}\right)=\left|c_{i}\right| \cdot \Delta_{i}$, so the range of $f$ is $[\widetilde{y}-\Delta, \widetilde{y}+\Delta]$, where $\Delta=\sum_{i=1}^{n}\left|c_{i}\right| \cdot \Delta_{i}$

Towards an algorithm. To compute $\Delta=\sum_{i=1}^{n}\left|c_{i}\right| \cdot \Delta_{i}$, we need to find $c_{i}$. If we replace one of $\widetilde{x}_{i}$ with $\widetilde{x}_{i}+\Delta_{i}$, then, due to linearization, we get

$$
y_{i} \stackrel{\text { def }}{=} f\left(\widetilde{x}_{1}, \ldots, \widetilde{x}_{i-1}, \widetilde{x}_{i}+\Delta_{i}, \widetilde{x}_{i+1}, \ldots, \widetilde{x}_{n}\right)=\widetilde{y}+c_{i} \cdot \Delta_{i} .
$$

Thus, $\left|c_{i}\right| \cdot \Delta_{i}=\left|y_{i}-\widetilde{y}\right|$ and hence $\Delta=\sum_{i=1}^{n}\left|y_{i}-\widetilde{y}\right|$.

Resulting algorithm. Compute $\widetilde{y}=f\left(\widetilde{x}_{1}, \ldots, \widetilde{x}_{n}\right)$, compute $n$ values $y_{i}=P_{f}\left(\widetilde{x}_{1}, \ldots, \widetilde{x}_{i-1}, \widetilde{x}_{i}+\Delta_{i}, \widetilde{x}_{i+1}, \ldots, \widetilde{x}_{n}\right)$, then compute $\Delta=\sum_{i=1}^{n}\left|y_{i}-\widetilde{y}\right|$ and $[\widetilde{P}-\Delta, \widetilde{P}+\Delta]$.

This algorithm requires $n+1$ calls to $f$ : to compute $\widetilde{y}$ and $n$ values $y_{i}$.

Towards a faster algorithm. When the number of inputs $n$ is large, $n+1$ calls may be too long. To speed up computations, we can use the following 
property of Cauchy distribution, with density $\$ \rho_{\delta}(x)=\frac{\delta}{\pi} \cdot \frac{1}{1+\frac{x^{2}}{\delta^{2}}}:$ if $\eta_{i}$ are independently Cauchy-distributed with parameters $\Delta_{i}$, then $\eta \stackrel{\text { def }}{=} \sum_{i=1}^{n} c_{i} \cdot \eta_{i}$ is Cauchy-distributed with parameter $\Delta=\sum_{i=1}^{c}\left|c_{i}\right| \cdot \Delta_{i}$.

Once we get simulated Cauchy-distributed values $\eta$, we can estimate $\Delta$ by the Maximum Likelihood method. We also need to scale $\eta_{i}$ to the interval $\left[-\Delta_{i}, \Delta_{i}\right]$ on which the linear approximation is applicable.

Resulting faster algorithm. ${ }^{\mathbf{7}}$ First, we compute $\widetilde{y}=f\left(\widetilde{x}_{1}, \ldots, \widetilde{x}_{n}\right)$. For some $N$ (e.g., 200), for $k=1, \ldots, N$, we repeatedly:

- use the random number generator to compute $r_{i}^{(k)}, i=1,2, \ldots, n$, uniformly distributed on $[0,1]$;

- compute Cauchy distributed values as $c_{i}^{(k)}=\tan \left(\pi \cdot\left(r_{i}^{(k)}-0.5\right)\right)$;

- compute the largest value $K$ of the values $\left|c_{i}^{(k)}\right|$;

- compute simulated "actual values" $x_{i}^{(k)}=\widetilde{x}_{i}+\frac{\Delta_{i} \cdot c_{i}^{(k)}}{K}$;

- apply $f$ and compute $\Delta y^{(k)}=K \cdot\left(f\left(x_{1}^{(k)}, \ldots, x_{n}^{(k)}\right)-\widetilde{y}\right)$.

Then, we compute $\Delta \in\left[0, \max _{k}\left|\Delta y^{(k)}\right|\right]$ by applying the bisection method to the equation $\frac{1}{1+\left(\frac{\Delta y^{(1)}}{\Delta}\right)^{2}}+\ldots+\frac{1}{1+\left(\frac{\Delta y^{(N)}}{\Delta}\right)^{2}}=\frac{N}{2}$. We stop when we get $\Delta$ with accuracy $\approx 20 \%$ (accuracy $1 \%$ and $1.2 \%$ is approximately the same).

The Cauchy-variate algorithm requires $N \approx 200$ calls to $f$. So, when $n \gg 200$, it is much faster than the above linearization-based algorithm.

\section{Beyond Linearization, Towards Interval Computations}

Linearizaion is sometimes not sufficient. In many application areas, it is sufficient to have an approximate estimate of $y$. However, sometimes, we need to guarantee that $y$ does not exceed a certain threshold $y_{0}$ : in nuclear engineering, the temperatures and the neutron flows should not exceed the critical values; a spaceship should land on the planet and does not fly past it, etc. 
The only way to guarantee this is to have an interval $\mathbf{Y}=[\underline{Y}, \bar{Y}]$ for which $\mathbf{y} \subseteq \mathbf{Y}$ and $\bar{Y} \leq y_{0}$. Such an interval is called an enclosure. Computing such an enclosure is one of the main tasks of interval computations.

Interval computations: a brief history. The origins of interval computations can be traced to the work of Archimedes from Ancient Greece who used intervals to bound values like $\pi$; see, e.g., ${ }^{1}$. Its modern revival was boosted by three pioneers: Mieczyslaw Warmus (Poland), Teruo Sunaga (Japan), and Ramon Moore (USA) in 1956-59. The first successful application was taking interval uncertainty into account when planning spaceflights to the Moon. Since then, there were many successful applications: to design of elementary particle colliders (Martin Berz, Kyoko Makino, USA), to checking whether an asteroid will hit the Earth (M. Berz, R. Moore, USA), to robotics (L. Jaulin, France; A. Neumaier, Austria), to chemical engineering (Marc Stadtherr, USA), etc. ${ }^{4,9}$.

Interval arithmetic: foundations of interval techniques. The problem is to compute the range

$$
[\underline{y}, \bar{y}]=\left\{f\left(x_{1}, \ldots, x_{n}\right) \mid x_{1} \in\left[\underline{x}_{1}, \bar{x}_{1}\right], \ldots, x_{n} \in\left[\underline{x}_{n}, \bar{x}_{n}\right]\right\} .
$$

For arithmetic operations $f\left(x_{1}, x_{2}\right)$ (and for elementary functions), we have explicit formulas for the range. For example, when $x_{1} \in \mathbf{x}_{1}=\left[\underline{x}_{1}, \bar{x}_{1}\right]$ and $x_{2} \in \mathbf{x}_{2}=\left[\underline{x}_{2}, \bar{x}_{2}\right]$, then:

- The range $\mathbf{x}_{1}+\mathbf{x}_{2}$ for $x_{1}+x_{2}$ is $\left[\underline{x}_{1}+\underline{x}_{2}, \bar{x}_{1}+\bar{x}_{2}\right]$.

- The range $\mathbf{x}_{1}-\mathbf{x}_{2}$ for $x_{1}-x_{2}$ is $\left[\underline{x}_{1}-\bar{x}_{2}, \bar{x}_{1}-\underline{x}_{2}\right]$.

- The range $\mathbf{x}_{1} \cdot \mathbf{x}_{2}$ for $x_{1} \cdot x_{2}$ is

$$
\left[\min \left(\underline{x}_{1} \cdot \underline{x}_{2}, \underline{x}_{1} \cdot \bar{x}_{2}, \bar{x}_{1} \cdot \underline{x}_{2}, \bar{x}_{1} \cdot \bar{x}_{2}\right), \max \left(\underline{x}_{1} \cdot \underline{x}_{2}, \underline{x}_{1} \cdot \bar{x}_{2}, \bar{x}_{1} \cdot \underline{x}_{2}, \bar{x}_{1} \cdot \bar{x}_{2}\right)\right] .
$$

The range $1 / \mathbf{x}_{1}$ for $1 / x_{1}$ is $\left[1 / \bar{x}_{1}, 1 / \underline{x}_{1}\right]$ (if $0 \notin \mathbf{x}_{1}$ ).

Straightforward interval computations. In general, we can parse an algorithm (i.e., represent it as a sequence of elementary operations) and then perform the same operations, but with intervals instead of numbers.

For example, to compute $f(x)=(x-2) \cdot(x+2)$, the computer first computes $r_{1}:=x-2$, then $r_{2}:=x+2$, and $r_{3}:=r_{1} \cdot r_{2}$. So, for estimating the range of $f(x)$ for $x \in[1,2]$, we compute $\mathbf{r}_{1}:=[1,2]-[2,2]=[-1,0]$, $\mathbf{r}_{2}:=[1,2]+[2,2]=[3,4]$, and $\mathbf{r}_{3}:=[-1,0] \cdot[3,4]=[-4,0]$.

Here, the actual range is $f(\mathbf{x})=[-3,0]$. This example shows that we need more efficient ways of computing an enclosure $\mathbf{Y} \supseteq \mathbf{y}$. 
First idea: use of monotonicity. For arithmetic, we had exact ranges, because,,+- . are monotonic in each variable, and monotonicity helps: if $f\left(x_{1}, \ldots, x_{n}\right)$ is (non-strictly) increasing $(f \uparrow)$ in each $x_{i}$, then $f\left(\mathbf{x}_{1}, \ldots, \mathbf{x}_{n}\right)=\left[f\left(\underline{x}_{1}, \ldots, \underline{x}_{n}\right), f\left(\bar{x}_{1}, \ldots, \bar{x}_{n}\right)\right]$. Similarly, if $f \uparrow$ for some $x_{i}$ and $f \downarrow$ for other $x_{j}$.

It is known that $f \uparrow$ in $x_{i}$ if $\frac{\partial f}{\partial x_{i}} \geq 0$. So, to check monotonicity, we can check that the range $\left[\underline{r}_{i}, \bar{r}_{i}\right]$ of $\frac{\partial f}{\partial x_{i}}$ on $\mathbf{x}_{i}$ has $\underline{r}_{i} \geq 0$. Here, differentiation can be performed by available Automatic Differentiation (AD) tools, an estimating ranges of $\frac{\partial f}{\partial x_{i}}$ can be done by using straightforward interval computations.

For example, for $f(x)=(x-2) \cdot(x+2)$, the derivatives is $2 x$, so its range on $\mathbf{x}=[1,2]$ is $[2,4]$, with $2 \geq 0$. Thus, we get the exact range $f([1,2])=[f(1), f(2)]=[-3,0]$.

Second idea: centered form. In the general non-monotonic case, we can use the general version of linearization - the Intermediate Value Theorem, according to which

$$
f\left(x_{1}, \ldots, x_{n}\right)=f\left(\widetilde{x}_{1}, \ldots, \widetilde{x}_{n}\right)+\sum_{i=1}^{n} \frac{\partial f}{\partial x_{i}}(\chi) \cdot\left(x_{i}-\widetilde{x}_{i}\right)
$$

for some $\chi_{i} \in \mathbf{x}_{i}$. Because of this theorem, we can conclude that $f\left(x_{1}, \ldots, x_{n}\right) \in \mathbf{Y}$, where

$$
\mathbf{Y}=\widetilde{y}+\sum_{i=1}^{n} \frac{\partial f}{\partial x_{i}}\left(\mathbf{x}_{1}, \ldots, \mathbf{x}_{n}\right) \cdot\left[-\Delta_{i}, \Delta_{i}\right]
$$

Here also, differentiation can be done by Automatic Differentiation (AD) tools, and estimating the ranges of derivatives can be done, if appropriate, by monotonicity, or else by straightforward interval computations, or also by centered form (this will take more time but lead to more accurate results).

Third idea: bisection. It is known that the inaccuracy of the first order approximation (like the ones we used) is $O\left(\Delta_{i}^{2}\right)$. So, when $\Delta_{i}$ is too large and the accuracy is low, we can split the corresponding interval in half (reducing the inaccuracy from $\Delta_{i}^{2}$ to $\Delta_{i}^{2} / 4$ ), and then take the union of the resulting ranges.

For example, the function $f(x)=x \cdot(1-x)$ is not monotonic for $x \in$ 
$\mathbf{x}=[0,1]$. So, we take $\mathbf{x}^{\prime}=[0,0.5]$ and $\mathbf{x}^{\prime \prime}=[0.5,1]$; on the 1st subinterval, the range of the derivative is $1-2 \cdot \mathbf{x}=1-2 \cdot[0,0.5]=[0,1]$, so $f \uparrow$ and $f\left(\mathbf{x}^{\prime}\right)=[f(0), f(0.5)]=[0,0.25]$. On the 2nd subinterval, we have $1-2 \cdot \mathbf{x}=1-2 \cdot[0.5,1]=[-1,0]$, so $f \downarrow$ and $f\left(\mathbf{x}^{\prime \prime}\right)=[f(1), f(0.5)]=[0,0.25]$. The resulting estimate is $f\left(\mathbf{x}^{\prime}\right) \cup f\left(\mathbf{x}^{\prime \prime}\right)=[0,0.25]$, which is the exact range.

These ideas underlie efficient interval computations algorithms and software packages $3,4,6,9$.

\section{Partial Information about Probabilities}

Formulation of the problem. In the ideal case, we know the probability distributions. In this case, in principle, we can find the distribution for $y=f\left(x_{1}, \ldots, x_{n}\right)$ by using Monte-Carlo simulations.

In the previous section, we considered situations when we only know an interval of possible values. In practice, in addition to the intervals, we sometimes also have partial information about the probabilities. How can we take this information into account?

How to represent partial information about probabilities. In general, there are many ways to represent a probability distribution; it is desirable to select a representation which is the most appropriate for the corresponding practical problem. In most practical problems, the ultimate objective is to make decisions. According to decision theory, a decision maker should look for an alternative $a$ that maximizes the expected utility $E_{x}[u(x, a)] \rightarrow \max _{a}$

When the utility function $u(x)$ is smooth, we can expand it in Taylor series $u(x)=u\left(x_{0}\right)+\left(x-x_{0}\right) \cdot u^{\prime}\left(x_{0}\right)+\ldots$; this shows that, to estimate $E[u]$, we must know moments. In this case, partial information means that we only have interval bounds on moments. There are known algorithms for processing such bounds; see, e.g., ${ }^{10}$.

Another case is when we have a threshold-type utility function $u(x)$ : e.g., for a chemical plant, drastic penalties start if the pollution level exceeds a certain threshold $x_{0}$. In this case, to find the expected utility, we need the know the values of the $\operatorname{cdf} F(x)=P(\xi \leq x)$. Partial information means that, for every $x$, we only have interval bounds $[\underline{F}(x), \bar{F}(x)]$ on the actual (unknown) cdf; such bounds are known as a $p$-box. There are also known algorithms for processing such boxes; see, e.g., ${ }^{2,10}$. 
Example of processing p-boxes. Suppose that we know p-boxes $\left[\underline{F}_{1}\left(x_{1}\right), \bar{F}_{1}\left(x_{1}\right)\right]$ and $\left[\underline{F}_{2}\left(x_{2}\right), \bar{F}_{2}\left(x_{2}\right)\right]$ for quantities $x_{1}$ and $x_{2}$, we do not have any information about the relation between $x_{1}$ and $x_{2}$, and we want to find the p-box corresponding $\underline{F}(y), \bar{F}(y)]$ corresponding to $y=x_{1}+x_{2}$.

It is known that for every two events $A$ and $B$,

$$
P(A \vee B)=P(A)+P(B)-P(A \& B) \leq P(A)+P(B) .
$$

In particular, $P(\neg A \vee \neg B) \leq P(\neg A)+P(\neg B)$. Here, $P(\neg A)=1-P(A)$, $P(\neg B)=1-P(B)$, and $P(\neg A \vee \neg B)=1-P(A \& B)$, thus, $1-P(A \& B) \leq$ $(1-P(A))+(1-P(B))$ and so, $P(A \& B) \geq P(A)+P(B)-1$. We also know that $P(A \& B) \geq 0$, hence $P(A \& B) \geq \max (P(A)+P(B)-1,0)$. Let us use this inequality to get the desired bounds for $F(y)$.

If $\xi_{1} \leq x_{1}$ and $\xi_{2} \leq x_{2}$, then $\xi \stackrel{\text { def }}{=} \xi_{1}+\xi_{2} \leq x_{1}+x_{2}$. Thus, if $x_{1}+x_{2}=$ $y$, then $F(y)=P(\xi \leq y) \geq P\left(\xi_{1} \leq x_{1} \& \xi_{2} \leq x_{2}\right)$. Due to the above inequality, $P\left(\xi_{1} \leq x_{1} \& \xi_{2} \leq x_{2}\right) \geq P\left(\xi \leq x_{1}\right)+P\left(\xi_{2} \leq x_{2}\right)-1$. Here, $P\left(\xi_{i} \leq x_{i} \geq \underline{F}_{i}\left(x_{i}\right)\right.$, so $F(y) \geq \underline{F}_{1}\left(x_{1}\right)+\underline{F}_{2}\left(x_{2}\right)-1$. Thus, as the desired lower bound $\underline{F}(y)$, we can take the largest of the corresponding right-hand sides: $\underline{F}(y)=\max \left(\max _{x_{1}, x_{2}: x_{1}+x_{2}=y}\left(\underline{F}_{1}\left(x_{1}\right)+\underline{F}_{2}\left(x_{2}\right)-1\right), 0\right)$, i.e.,

$$
\underline{F}(y)=\max \left(\max _{x_{1}}\left(\underline{F}_{1}\left(x_{1}\right)+\underline{F}_{2}\left(y-x_{1}\right)-1\right), 0\right) .
$$

To find the upper bound for $F(y)$, let us find a similar lower bound for $1-F(y)=P(\xi>y)$. If $x_{1}+x_{2}=y, \xi_{1}>x_{1}$, and $\xi_{2}>x_{2}$, then $\xi=\xi_{1}+\xi_{2}>y$. Here, $P\left(\xi_{i}>x_{i}\right)=1-P\left(\xi_{i} \leq x_{i}\right)=1-F_{i}\left(x_{i}\right)$. Thus,

$$
\begin{aligned}
1-F(y) & =P(\xi>y) \geq P\left(\xi_{1}>x_{1} \& \xi_{2}>x_{2}\right) \geq P\left(\xi_{1}>x_{1}\right)+P\left(\xi_{2}>x_{2}\right)-1 \\
& =\left(1-F_{1}\left(x_{1}\right)\right)+\left(1-F_{2}\left(x_{2}\right)\right)-1=1-F_{1}\left(x_{1}\right)-F_{2}\left(x_{2}\right),
\end{aligned}
$$

hence $F(y) \leq F_{1}\left(x_{1}\right)+F_{2}\left(x_{2}\right)$. Since $F_{i}\left(x_{i}\right) \leq \bar{F}_{i}\left(x_{i}\right)$, we have $F(y) \leq$ $\bar{F}_{1}\left(x_{1}\right)+\bar{F}_{2}\left(x_{2}\right)$. Thus, as the desired upper bound $\bar{F}(y)$, we can take the smallest of the corresponding right-hand sides:

$$
\begin{gathered}
\bar{F}(y)=\min \left(\min _{x_{1}, x_{2}: x_{1}+x_{2}=y}\left(\bar{F}_{1}\left(x_{1}\right)+\bar{F}_{2}\left(x_{2}\right)\right), 1\right), \text { i.e. } \\
\bar{F}(y)=\min \left(\min _{x_{1}}\left(\bar{F}_{1}\left(x_{1}\right)+\bar{F}_{2}\left(y-x_{1}\right)\right), 1\right) .
\end{gathered}
$$

Similar formulas can be derived for other elementary operations. 
How to represent p-boxes. Representing a p-box means representing two cdfs $\underline{F}(x)$ and $\bar{F}(x)$. For each cdf $F(x)$, to represent all its values with accuracy $\frac{1}{n}$, it is sufficient to store $n-1$ quantiles $x_{1}<\ldots<x_{n-1}$, i.e., values $x_{i}$ for which $F\left(x_{i}\right)=\frac{i}{n}$. These values divide the real line into segments $\left[x_{i}, x_{i+1}\right]$, where $x_{0} \stackrel{\text { def }}{=}-\infty$ and $x_{n+1} \stackrel{\text { def }}{=}+\infty$.

Each real value $x$ belongs to one of these segments $\left[x_{i}, x_{i+1}\right]$, in which case, due to monotonicity of $F(x)$, we have $F\left(x_{i}\right)=\frac{i}{n} \leq F(x) \leq \frac{i+1}{n}=$ $F\left(x_{i+1}\right)$, hence $\left|F(x)-\frac{i}{n}\right| \leq \frac{1}{n}$.

Need to go beyond p-boxes. In many practical situations, we need to maintain the value within a certain interval: e.g., the air conditioning must maintain the temperature within certain bounds, a spaceship must land within a certain region, etc. In such cases, the utility drastically drops if we are outside the interval; thus, the expected utility is proportional to the probability $F(a, b)=P(\xi \in(a, b])$ to be within the corresponding interval $(a, b]$. In such situations, partial information about probabilities means that for $a$ and $b$, we only know the interval $[\underline{F}(a, b), \bar{F}(a, b)]$ containing the actual (unknown) values $F(a, b)$.

When we know the exact cdf $F(x)$, then we can compute $F(a, b)$ as $F(a)-F(b)$. However, in case of partial information, it is not sufficient to only know the p-box. For example, let us assume that $x$ is uniformly distributed on some interval of known width $\varepsilon>0$, but we do not know on which. In this case, as one can easily see, for every $x, \underline{F}(x)=0$ and $\bar{F}(x)=1$ - irrespective on $\varepsilon$. On the other hand, for any interval $[a, b]$, we have $\bar{F}(a, b)=\min \left(\frac{b-a}{\varepsilon}, 1\right)$. This bound clearly depends on $\varepsilon$ and thus, cannot be uniquely determined by the p-box values.

How to process this more general information. Good news is that we process this more general information similarly to how we process p-boxes.

Specifically, when $\xi_{1} \in \mathbf{x}_{1}=\left(\underline{x}_{1}, \bar{x}_{1}\right]$ and $\xi_{2} \in \mathbf{x}_{2}=\left(\underline{x}_{2}, \bar{x}_{2}\right]$, then $\xi=\xi_{1}+\xi_{2} \in \mathbf{x}_{1}+\mathbf{x}_{2}=\left(\underline{x}_{1}+\underline{x}_{2}, \bar{x}_{1}+\bar{x}_{2}\right]$. Thus, if $\mathbf{x}_{1}+\mathbf{x}_{2} \subseteq \mathbf{y}=[\underline{y}, \bar{y}]$, we have

$$
\begin{gathered}
F(\underline{y}, \bar{y}) \geq P\left(\xi_{1} \in \mathbf{x}_{1} \& \xi_{2} \in \mathbf{x}_{2}\right) \geq P\left(\xi_{1} \in \mathbf{x}_{1}\right)+P\left(\xi_{2} \in \mathbf{x}_{2}\right)-1 \geq \\
\underline{F}_{1}\left(\mathbf{x}_{1}\right)+\underline{F}_{2}\left(\mathbf{x}_{2}\right)=1 .
\end{gathered}
$$


So, as the desired lower bound $\underline{F}(\underline{y}, \bar{y})$, we can take the largest of the corresponding right-hand sides:

$$
\underline{F}(\underline{y}, \bar{y})=\max \left(\max _{\mathbf{x}_{1}, \mathbf{x}_{2}: \mathbf{x}_{1}+\mathbf{x}_{2} \subseteq \mathbf{y}}\left(\underline{F}_{1}\left(\mathbf{x}_{1}\right)+\underline{F}_{2}\left(\mathbf{x}_{2}\right)-1\right), 0\right) .
$$

This formula is very similar to the formula for p-boxes. The formula for the upper bound comes from the fact that $F(y, \bar{y})=F(\bar{y})-F(y)$, and thus, $F(\underline{y}, \bar{y}) \leq \bar{F}(\bar{y})-\underline{F}(\underline{y})$. We already know the values $\bar{F}(\bar{y})-\underline{F}(\underline{y})$, thus we can take their difference as the desired upper bound $\bar{F}(\underline{y}, \bar{y})$ :

$$
\begin{gathered}
\bar{F}(\underline{y}, \bar{y})=\min \left(\min _{x_{1}}\left(\bar{F}_{1}\left(x_{1}\right)+\bar{F}_{2}\left(\bar{y}-x_{1}\right)\right), 1\right)- \\
\max \left(\max _{x_{1}}\left(\underline{F}_{1}\left(x_{1}\right)+\underline{F}_{2}\left(y-x_{1}\right)-1\right), 0\right) .
\end{gathered}
$$

Similar formulas can be obtained for other elementary operations.

How to represent this more general information. Not so good news is that representing such a more general information is much more difficult than representing p-boxes.

Indeed, similarly to p-boxes, we would like to represent all the values $\underline{F}(a, b)$ and $\bar{F}(a, b)$ with a given accuracy $\frac{1}{n}$, i.e., we would like to find the values $x_{1}<\ldots<x_{N}$ for which $x_{i} \leq a \leq x_{i+1}$ and $x_{j} \leq b \leq x_{j+1}$ implies $\underline{F}(a, b)-\underline{F}\left(x_{i}, x_{j}\right) \leq \frac{1}{n}$ and $\mid \bar{F}(a, b)-\bar{F}\left(x_{i}, x_{j}\right) \leq \frac{1}{n}$.

For p-boxes, we could use $N=n$ values $x_{i}$. Let us show that for the bounds on $P(a, b)$, there is no upper bound on the number of values needed. Namely, we will show that in the above example, when $\varepsilon \rightarrow 0$, the corresponding number of points $N$ grows indefinitely: $N \rightarrow \infty$. Indeed, when $j=i, a=x_{i}$, and $b=x_{i+1}$, then, due to $\bar{F}\left(x_{i}, x_{i}\right)=0$, the above condition means $\bar{F}\left(x_{i}, x_{i+1}\right) \leq \frac{1}{n}$. Thus, we must have $\frac{x_{i+1}-x_{i}}{\varepsilon} \leq \frac{1}{n}$, i.e., $x_{i+1}-x_{i} \leq \frac{\varepsilon}{n}$. The next point $x_{i+1}$ is this close to the previous one, so, e.g., on the unit interval $[0,1]$, we need at least $N \geq \frac{n}{\varepsilon}$ such points. When $\varepsilon \rightarrow 0$, the number of such points indeed tends to infinity.

It is worth mentioning that we can have an upper bound on $N$ if we know an upper bound $d$ on the probability density $\rho(x)$ : in this case, $F(a, b) \leq(b-a) \cdot d$ and thus, to get the desired accuracy $\frac{1}{n}$, it is sufficient to have $x_{i+1}-x_{i}=\frac{1}{n \cdot d}$. On an interval of width $W$, we thus need 
$N=W x_{i+1}-x_{i}=W \cdot n \cdot d$ points.

\section{Acknowledgments}

This work was supported in part by the National Science Foundation grants HRD-0734825 and HRD-1242122 (Cyber-ShARE Center of Excellence) and DUE-0926721. The author is greatly thankful to Scott Ferson, to Franco Pavese, and to all the participants of the International Conference on Advanced Mathematical and Computational Tools in Metrology and Testing AMTCM'2014 (St. Petersburg, Russia, September 9-12, 2014) for valuable discussions.

\section{References}

1. Archimedes, On the measurement of the circle, In: T. L. Heath (ed.), The Works of Archimedes (Dover, New York, 1953).

2. S. Ferson et al., Constructing Probability Boxes and Dempster-Shafer Structures (Sandia Nat'l Labs, Report SAND2002-4015, 2003).

3. Interval computations website http://www.cs.utep.edu/interval-comp

4. L. Jaulin et al., Applied Interval Analysis (Springer, London, 2001).

5. E. T. Jaynes and G. L. Bretthorst, Probability Theory: The Logic of Science (Cambridge University Press, Cambridge, UK, 2003).

6. V. Kreinovich, Interval computations and interval-related statistical techniques, In: F. Pavese and A. B. Forbes (eds.), Data Modeling for Metrology and Testing in Measurement Science (Birkhauser-Springer, Boston, 2009), pp. 117-145.

7. V. Kreinovich and S. Ferson, A new Cauchy-Based black-box technique for uncertainty in risk analysis, Reliability Engineering and Systems Safety 85(1-3), 267-279 (2004).

8. V. Kreinovich et al., Computational Complexity and Feasibility of Data Processing and Interval Computations (Kluwer, Dordrecht, 1997).

9. R. E. Moore, R. B. Kreinovich, and M. J. Cloud, Introduction to Interval Analysis (SIAM Press, Philadelphia, Pennsylvania, 2009).

10. H. T. Nguyen et al., Computing Statistics under Interval and Fuzzy Uncertainty (Springer, Berlin, Heidelberg, 2012).

11. S. G. Rabinovich, Measurement Errors and Uncertainty:Theory and Practice (Springer, Berlin, 2005). 